\title{
Conceptual Design for Consolidation TCAP
}

by

J. E. Klein

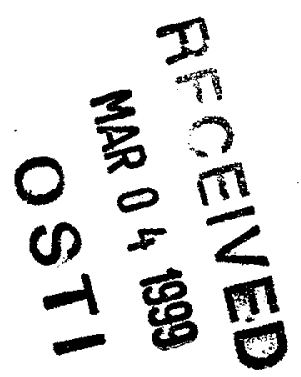

Westinghouse Savannah River Company

Savannah River Site

Aiken, South Carolina 29808

L. K. Heung

J. H. Scogin

D. W. Howard

M. W. Lee

T. Motyka

A. S. Poore

This paper was prepared in connection with work done under the above contract number with the U.S.

Department of Energy. By acceptance of this paper, the publisher and/or recipient acknowledges the U.S. Government's right to retain a nonexclusive, royalty-free license in and to any copyright covering this paper, along with the right to reproduce and to authorize others to reproduce all or part of the copyrighted paper. 


\section{DISCLAIMER}

This report was prepared as an account of work sponsored by an agency of the United States Government. Neither the United States Government nor any agency thereof, nor any of their employees, makes any warranty, express or implied, or assumes any legal liability or responsibility for the accuracy, completeness, or usefulness of any information, apparatus, product, or process disclosed, or represents that its use would not infringe privately owned rights. Reference herein to any specific commercial product, process, or service by trade name, trademark, manufacturer, or otherwise does not necessarily constitute or imply its endorsement, recommendation, or favoring by the United States Government or any agency thereof. The views and opinions of authors expressed herein do not necessarily state or reflect those of the United States Government or any agency thereof.

This report has been reproduced directly from the best available copy.

Available to DOE and DOE contractors from the Office of Scientific and Technical Information, P. O. Box 62, Oak Ridge, TN 37831; prices available from (423) 576-8401.

Available to the public from the National Technical Information Service, U. S. Department of Commerce, 5285 Port Royal Road, Springfield, VA 22161. 


\section{DISCLAIMER}

Portions of this document may be illegible in electronic image products. Images are produced from the best available original document. 


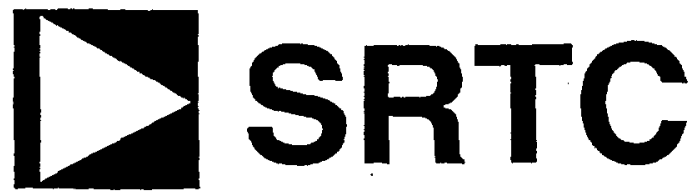

\section{Savannah River Technology Center}

Chemical and Hydrogen Technology Section

WSRC-TR-97-00340TL

October 24, 1997

CC:

\begin{tabular}{ll} 
T. Motyka & 773-A \\
A. F. Riechman & 773-A \\
D. M. Hasty & 232-H \\
L. K. Heung & $773-\mathrm{A}$ \\
J. E. Klein & $773-\mathrm{A}$ \\
J. H. Scogin & $773-\mathrm{A}$ \\
A.S. Poore & $773-\mathrm{A}$ \\
J.S. Holder & 232-H \\
D. W. Howard & $773-\mathrm{A}$ \\
STI Files (4) & $703-43 \mathrm{~A}$ \\
\hline CHTS Files & $773-\mathrm{A}$
\end{tabular}

T. G. Ballweg,

$730-1 \mathrm{~B}$

H. T. Harris, $\quad 233-6 \mathrm{H}$

T. Osmundsen 730-1B

R. L. Rabun 235-H

E. A. Clark 773-A

M. A. Reffett $235-2 \mathrm{H}$

M. G. Scott 773-A

R. H. Hsu 773-A

G.S. Nichols 773-A

M.W. Lee 773-41A

TO: $\quad$ R. D. Buley $\quad 235-\mathrm{H}$

FROM: J.R. Knight 773-A

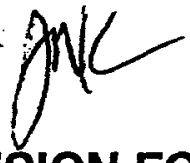
CONCEPTUAL DESIGN FOR CONSOLIDATION TCAP

A task team formed by Ted Motyka has developed two conceptual designs for the Isotope Separation System (ISS) of Tritium Facility Modernization and Consolidation (TFM\&C) project. A design similar to the Thermal Cycling Absorption Process (TCAP) units in the Replacement Tritium Facility (RTF) is recommended for the TFM\&C project; the other design has been designated as backup for the TFM\&C project and as a potential replacement for the exiting RTF units.

Some development on the selected design is required, especially to confirm heating and cooling rates. Confirmation of throughput and separation ability will be performed using the backup design in a joint effort with Los Alamos National Laboratory. 


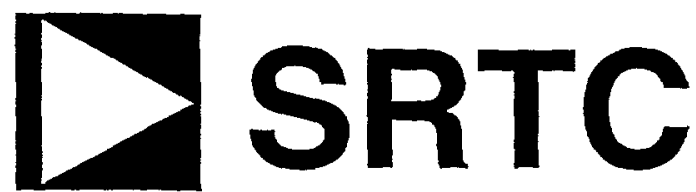

\title{
Savannah River Technology Center
}

Chemical and Hydrogen Technology Section

\section{CONCEPTUAL DESIGN FOR CONSOLIDATION TCAP}

\author{
J. E. Klein (Editor), L. K. Heung, D. W. Howard, M. W. Lee, T. Motyka, A. S. Poore, and J. H. Scogin \\ Savannah River Technology Center
}

\section{SUMMARY}

Two alternate Thermal Cycling Absorption Process (TCAP) designs have been developed for the Tritium Facility Modernization and Consolidation (TFM\&C) Project. The alternate designs were developed to improve upon the existing Replacement Tritium Facility (RTF) TCAP design and to eliminate the use of building distributed hot and cold nitrogen system.

A brief description of TCAP theory and modeling is presented, followed by an overview of the design criteria for the Isotope Separation System (ISS). Both designs are described in detail, along with a generic description of the complete TCAP system.

A design is recommend for the Consolidation Project, and a development plan for both designs is proposed.

\section{INTRODUCTION/BACKGROUND}

The Tritium Facility Modernization and Consolidation (TFM\&C) Project, or Consolidation Project, is being implemented to modernize and consolidate tritium gas processing into one facility. The Consolidation Project will replace needed tritium gas processing operations performed in the Tritium Facility's extraction building into the expansion area in the Replacement Tritium Facility (RTF).

Currently, isotopic separations are done by the RTF Thermal Cycling Absorption Process (TCAP) and by the extraction building's batch cryogenic distillation column. The RTF TCAPs perform deuterium/tritium (D-T) separations. One of the major isotopic separation operations performed by the cryogenic still is protium enrichment, defined here as the stripping of tritium from "low" tritium content gas streams for discharge to the atmosphere. Tritium enrichment, defined as the reduction of $\mathrm{D} 2$ and protium (H2) in "high" tritium content gas streams, can also be performed by the cryogenic still along with other isotopic separations (e.g. H-D, H-T, D-T).

For the Consolidation Project, the TCAP was chosen for the Isotope Separation System (ISS). The current RTF TCAP design uses thermal swings supplied by the RTF distributed hot and cold (H\&C) nitrogen systems, however, the Consolidation Project will not use the existing RTF heating and cooling (H\&C) systems for the ISS TCAPs. Furthermore, the use of the same type of H\&C systems

\section{Classification: Unclassified}

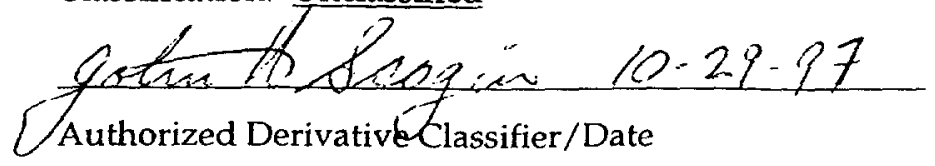


for the project is undesirable. The Chemical \& Hydrogen Technology Section (CHTS) Process Technology Group (PTG), initiated the task of developing a conceptual design for Consolidation TCAPs that do not use the existing RTF H\&C technology.

On May 5, 1997, Ted formed the TCAP Task Team was formed to examine alternative TCAP designs and develop a conceptual design of a TCAP-based ISS to meet the Consolidation Project requirements. One deliverables from this task team is this Conceptual Design Report with a target delivery date of $9 / 30 / 97$. Jim Klein was assigned team leader/facilitator. Core task team members were Kit Heung, Dave Howard, Myung Lee, George Nichols, Anita Poore, and John Scogin. Resource personnel included Elliot Clark and John Mickalonis of the Materials Technology Section (MTS), plus others.

The conceptual design process focused on evaluating alternatives for $\mathrm{H} \& \mathrm{C}$ TCAP columns due to the short development time available. The development path consisted of brainstorming on $\mathrm{H} \& \mathrm{C}$ alternatives, reducing the number of TCAP designs for development to two, holding a PreConceptual Design Review where non-team members evaluated the two TCAP concepts, and issuing this report. Each of these steps will be briefly described below.

Brainstorming sessions were used to identify H\&C alternatives and their applicability to the TCAP. Criteria for consideration included minimization of radioactive waste and tritiated organics formed, material hazards and incompatibility for use in gloveboxes, and ability to provide continuos heating or cooling to the TCAP. Phase change methods were considered too limited in capacity on a practical basis due to a fixed amount of heat transfer material available for $\mathrm{H} \& \mathrm{C}$ operations. $\mathrm{A}$ matrix of heating-by-cooling alternatives was generated and incompatible $\mathrm{H} \& \mathrm{C}$ combinations eliminated. It was assumed that "aluminum foam" will be used in the process side of all TCAP column designs to increase heat transfer rates and reduce cycle times.

Out of the task team meetings, two TCAP designs emerged: the Improved Stainless Steel Coil TCAP (ITCAP) and the Rectangular Array of Bored-In Tubes (RABIT) TCAP. Both designs were presented to a Pre-Conceptual Design Review Team for evaluation. The Review Team consisted of Jim Klein as the non-voting Chairman; Anita Poore as the non-voting recorder; Tom Ballweg from Consolidation Design Engineering, Bob Rabun from Tritium Technology, and Mark Reffett from Tritium Operations to represent the interests of Tritium Projects, Tritium Engineering, Tritium Operations, respectively; Elliot Clark from SRTC/MTS to provide a materials compatibility and fabricability perspective; Bob Hsu from SRTC/PTG to provide perspective on SRTC R\&D cycles; and Jeff Holder from SRTC Weapons Technology Group to balance the Review Team with equal SRTC and non-SRTC members. In the event of a tie vote, Ted Motyka as the responsible manager, in conjunction with Consolidation Project Management would select the ISS TCAP design.

The results of the Pre-Conceptual Design Review Team evaluation were three member voting for the ITCAP and three members voting for the RABIT TCAP. Using a numerical rating (with 576 point maximum) to rate how well each design met the design criteria selected for the evaluation process, the ITCAP received 299 points (51.9\%) while the RABIT TCAP 280 points (48.6\%). Due to the closeness of the voting and scoring, along with other research and development considerations, both designs are recommended for further development following a program outlined later in this report. The ITCAP was chosen as the base-line design for Consolidation, while the RABIT TCAP chosen as the back-up design and a candidate for replacing RTF TCAPs.

This Conceptual Design Report was written by members of the TCAP Task Team, with Jim Klein as editor. The report structure includes: 1) a description of the over-all TCAP design basis and modeling as background information on TCAP, 2) references to the Requirements/Design Criteria for the Consolidation Project ISS, 3) a description of the ITCAP, 4) a description of the RABIT TCAP, 5) a generic TCAP System Description, 6) required TCAP interfaces and services, and 7) recommendations for the Consolidation Project research and development program. 


\section{TCAP OVERALl DEsign BASIS AND MOdeL}

\section{BACKGROUND}

Hydrogen has three isotopes, protium, deuterium, and tritium, which are very similar in chemical properties. Isotope separation must be based on physical properties and is therefore more difficult to perform than chemical separation. TCAP, the Thermal Cycling Absorption Process, is a technique to separate mixtures of hydrogen into component isotopes. The process portion of the TCAP system consists of a column packed with palladium deposited on kieselguhr $(\mathrm{Pd} / \mathrm{k})$ and a plug-flow-reverser (PFR) packed with plain kieselguhr. Kieselguhr, a form of diatomaceous earth, has a low pressure drop for gas flows in the column and provides a large surface area for deposition of the palladium metal. The thin layer of palladium on kieselguhr readily absorbs hydrogen gas.

Palladium can absorb hydrogen isotopes to form metal hydrides. An isotherm is the constant temperature relationship between the equilibrium pressure and hydrogen concentration in the metal hydride (in atomic ratio of hydrogen to metal, $\mathrm{H} / \mathrm{M}, \mathrm{D} / \mathrm{M}$ or $\mathrm{T} / \mathrm{M}$ ). At constant pressure, metal hydrides absorb more hydrogen at lower temperatures than at higher temperatures. The separation factor is defined by the ratio of the heavier-to-lighter isotopic concentration ratio in the gas phase to the same ratio in the solid phase. Palladium preferentially absorbs the lighter isotope over the heavier isotope, which gives a separation factor larger than one. Palladium is an unique hydride in that it has a large isotope effect and its separation factor is larger at lower temperatures than at higher temperatures. The separation factor is some times called the "single stage " separation factor. A packed column can have many stages. The overall enrichment factor of a given column is roughly the single stage separation factor raised to the power of the number of stages. Therefore, larger separation factors and longer columns (more stages) will give better separation.

\section{Thermal Cycling Absorption Process Description}

TCAP operates on the principle similar to a gas chromatograph using a palladium column, but in a semi-continuous manner. One end of the column is dead-ended with the other end connected to the PFR. The hydrogen feed for isotopic separation is added at a fixed location on the column, a heavy isotope enriched stream is withdrawn at the dead end of the column (Product end), and a heavy isotope depleted stream is withdrawn at the end of the column where it is connected to the PFR (Raffinate end).

The column is thermally cycled by a H\&C system. During the heating cycle, the hydrogen gas is desorbed from palladium and the pressure in the column increases. Then, the desorbed gas is transferred into the PFR. During the cooling cycle, the hydrogen is absorbed into the palladium and the column pressure decreases which transfers the gas from the PFR back into column. As the gas flows through the column, isotopic exchange occurs between the gas and the solid (Pd) phases. Due to the isotope affect, the heavier isotope is released from the solid and migrates toward to the dead end of the column. In this manner, the heavier isotope is enriched at the dead end of the column and depleted at the PFR end of the column.

During every cooling/absorption cycle, a separation takes place with the lighter isotope preferentially absorbing into the palladium. During every heating/desorption cycle, some of the separation gained during the cooling cycle is lost. Since the separation factor for palladium is greater at colder temperatures than warmer temperatures, a net separation is obtained after each complete absorptiondesorption cycle. This net separation is what produces the Product and Raffinate purities.

In total reflux mode, gas is moving back and forth between the column and the PFR during H\&C cycles with no feed or gas withdrawal. After about 40 cycles, a relatively sharp concentration boundary is formed in the column and with high purity isotopes at both ends of the column. In production mode operation, a small fraction of the mixture is fed to the column (relative to the total 
column inventory of hydrogen isotopes) each cycle and the same amount removed from column as Product and Raffinate. The "cut" is the fraction of the Product stream to the total gas withdraw and is determined by the concentration at the feed point of the column.

\section{Design Considerations}

TCAP throughput capacity is proportional to the total column inventory of hydrogen isotopes and is inversely proportion to the cycle time. Column inventory is directly proportion to column length and cross-section area of the column. Doubling the diameter of the column would increase the inventory by a factor of four. Cycle time is the total time to heat and cool a column, transfer gas to and from the PFR, feed gas to the column, and withdraw product and raffinate from the column.

The purity of the Product and Raffinate depends mainly on the column length (the number of the stages in the column), and other factors such as the magnitude of the temperature swing, the absolute temperatures for the temperature swing, the throughput/feed rate, and the feed concentration. General trends are the longer the column, the higher the purity; the smaller the feed rate, the higher the purity; the larger the temperature swing, the higher the purity; the colder the lower temperature of the thermal swing, the higher the purity. Column length has a practical limit for TCAP operations since the longer the column, the higher the pressure drop in the column and thus lengthening gas transfer times to and from the PFR thus increasing cycle time.

The separation factor of protium to tritium is much larger than that of deuterium to tritium. A shorter column can achieve the same product purity for a tritium-protium separation than for tritium-deuterium separation at the same feed rate in a longer column. Conversely, for a fixed column length, the feed rate can be larger for a tritium-protium separation than a tritium-deuterium separation.

\section{MOdELS AND SimULATION}

SRTC has developed mathematical models and computer simulation programs for TCAP operation. Relationships between column size, cold and hot temperatures, and feed rate to Product/Raffinate purities can be calculated. This simulation package is a valuable resource for design calculations and optimization of the column's operation; however, the program has not been bench-marked and its results are not quantitative. The model is useful in predicting trends as functions of the various parameters, but not in calculating absolute column isotope purities or column processing rates.

To illustrate modeling results, the Raffinate impurities and Product impurities (expressed as one minus the Product mole fraction) for D-T and H-T separations as a function of the feed rate are calculated and shown in Figure 1 and Figure 2. The results are for a fixed column length for two different temperature swings: $+25^{\circ} \mathrm{C}$ to $125^{\circ} \mathrm{C}$ and $-25^{\circ} \mathrm{C}$ to $125^{\circ} \mathrm{C}$. The results show the trend of the impurity levels in the Product and Raffinate streams with temperature swing, feed rate, and the difference between $H / T$ and $D / T$ separations. Other variables fixed for the simulation were the feed location and the feed concentration. For engineering reasons, there will be limitations on the high and low temperatures for the TCAP operation.

The temperature dependence of the separation factor does have an impact on Product and Raffinate Purities. For a fixed difference in the temperature swing range, a TCAP column cycling between -25 to $125^{\circ} \mathrm{C}$ will give higher Product and Raffinate purities (or a larger feed rate) than the same column operation over the temperature range of +25 to $175^{\circ} \mathrm{C}$. Both columns operate over the same $150^{\circ} \mathrm{C}$ temperature swing, but the separation factor is much larger at the lower temperatures and gives better separation for fixed column operations.

Based on the given requirements such as throughput capacity and the purity of the Product and Raffinate streams, design parameters such as column length, column diameter, feed location, hot and cold temperatures of the column can be estimated using this program. Once a system is built, control parameters can be adjusted, such as feed rate, to determine the column's operating domain. 


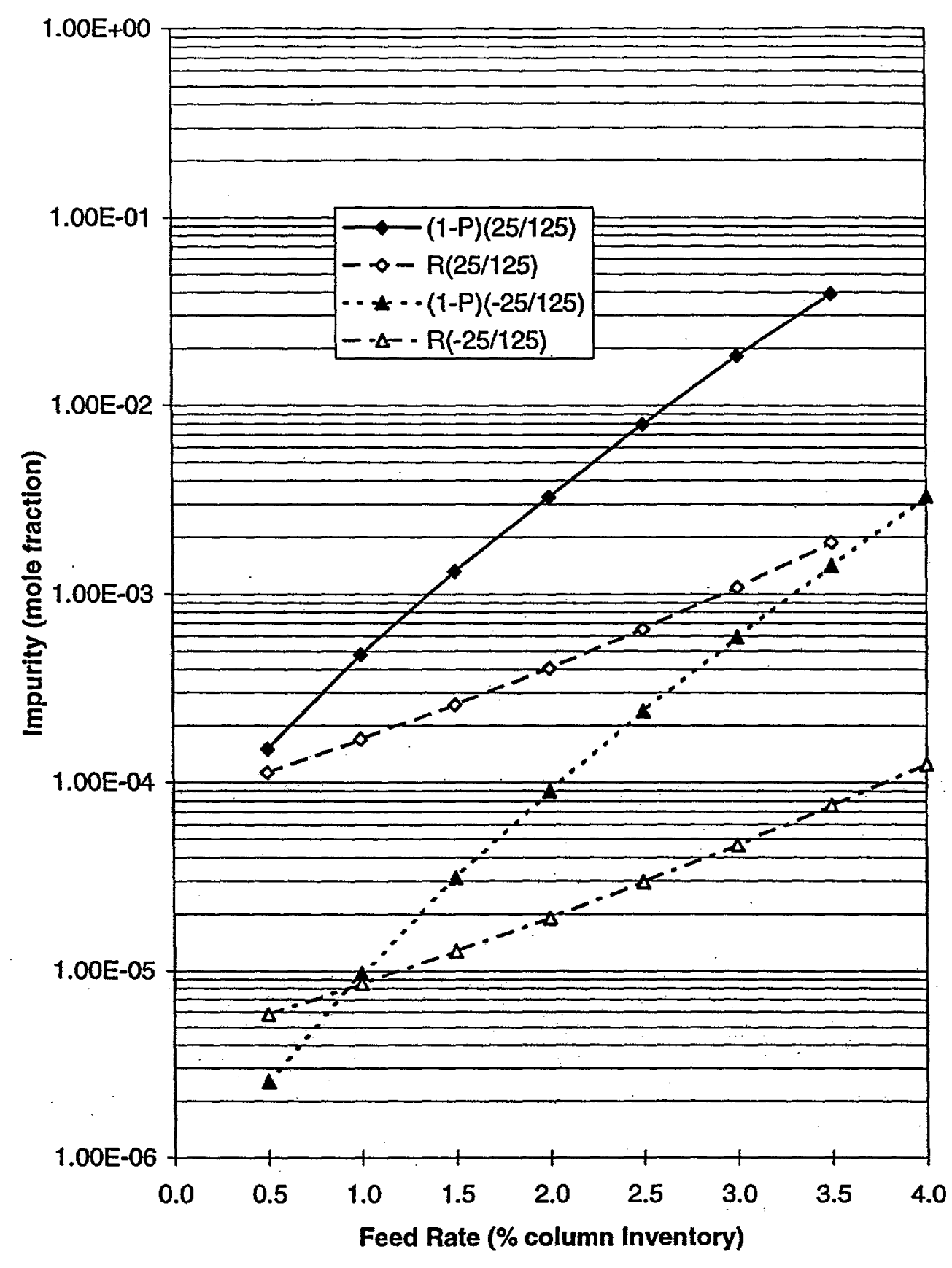

Figure 1. Impurity in Product/Raffinate vs. Feed Rate for D-T Separation 


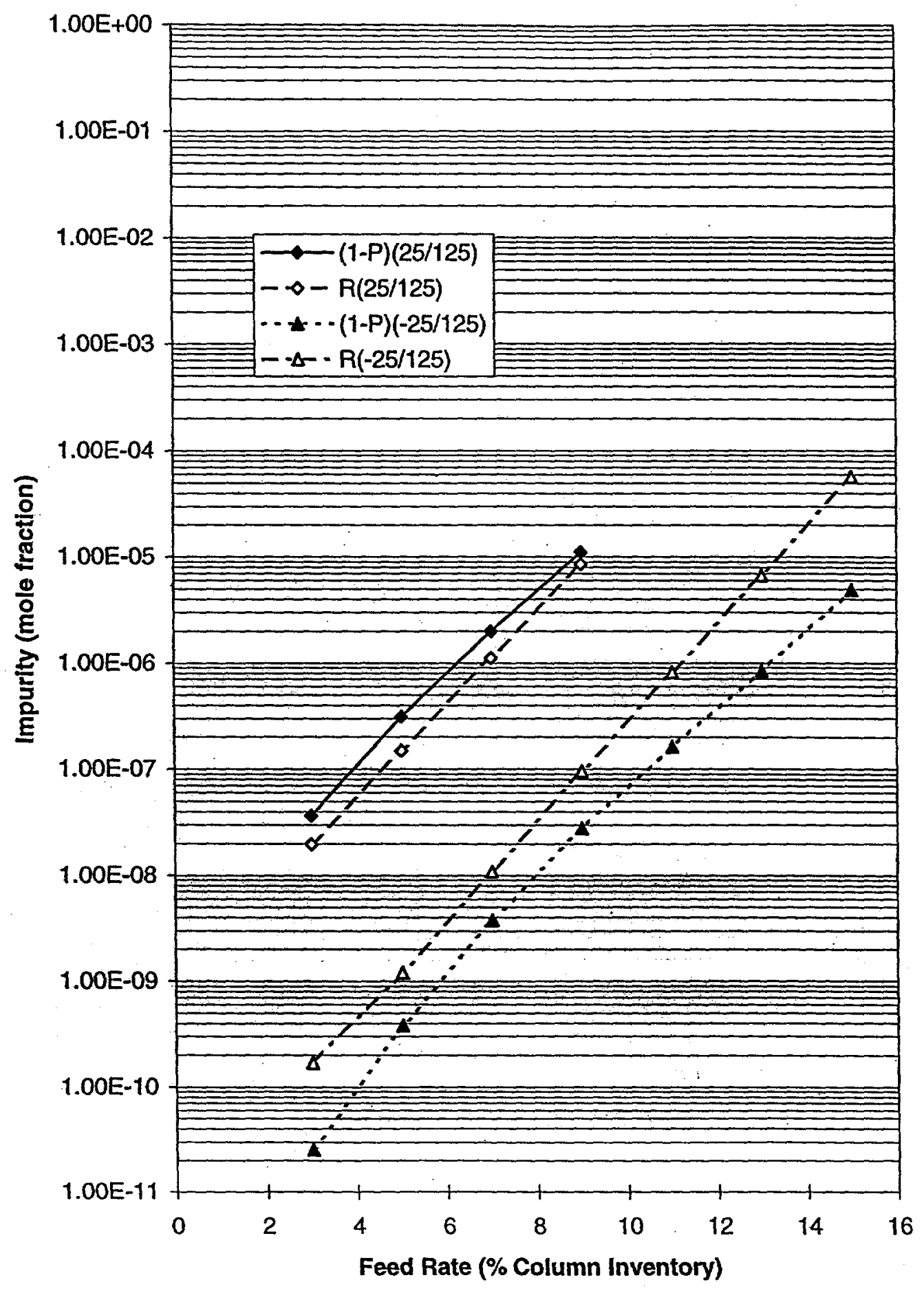

Figure 2. Impurity in Product/Raffinate vs. Feed Rate for H-T Separation 


\section{Consolidation tCaP Requirements/Design Criteria}

The Consolidation Project's proposal for the ISS design has two TCAP columns performing the tritium enrichment function and two TCAP columns performing protium enrichment function. The dividing line between "low" and "high" tritium content gas for isotopic separation has been set at nominally two mole percent tritium. The Consolidation ISS design is different from that recommended in the TCAP System Description of this report in hydride bed layout and column connections: the configuration in this report allows greater flexibility of operation of the Consolidation TCAP columns.

Detailed Consolidation ISS Requirements and Criteria are covered in a separate document [Ref. 1]. The separation gas load for protium enrichment is expected to be many times greater than that for tritium enrichment. On this basis, the designs discussed in this document will focus on TCAP designs sized for the protium enrichment function. A basis of 510,000 STP-L per year hydrogen isotope feed and a tritium concentration in the effluent protium stream of $5 \mathrm{ppm}$ will be used for these discussions. The Alternate TCAP designs for this separation function will be described below.

\section{ALTERNATIVETCAP DESCRIPTION: IMPROVED TCAP (ITCAP) DESCRIPTION}

The ITCAP builds upon the success of the RTF design, but makes improvements over the existing design. A schematic of the ITCAP system is shown in Figure 3. A summary and description of the advantages and disadvantages of using the ITCAP are summarized below:

\section{ITCAP SUMMARY}

- Builds on present demonstrated system for improvement.

- Closely couples the H\&C equipment and the column to minimize the length of the H\&C lines, and to eliminate the H\&C lines from the occupied area.

- Greatly reduces H\&C nitrogen pressures from existing pressures to about 1 atmosphere which permits the use of blowers instead of compressors, and, minimizes the potential for nitrogen leaks.

- Raises the cold temperature to permit the use of a single stage Freon compressor instead of the present 2-stage compressor.

- Uses routine purge of the H\&C nitrogen to limit the tritium level in the nitrogen system.

- Adds aluminum foam to the $\mathrm{Pd} / \mathrm{k}$ column to improve heat transfer.

- Considers aluminizing the surface of the stainless steel column to reduce tritium permeation through the column wall.

- Considers the use of thermal insulation on the inside of the column's jacket to reduce the heat load of the system.

\section{ADVANTAGES OF THEITCAP}

- Applies demonstrated technology to save project time and cost.

- Eliminates key undesired features of the present system which includes high pressure H\&C nitrogen lines in occupied areas, tritium buildup in the high pressure nitrogen, large nitrogen compressors, and large 2-stage Freon compressors.

- Improves internal heat transfer of column through the use of aluminum foam.

- Reduces tritium permeation through aluminum coating of the column.

\section{DisadVANTAGES OF THEITCAP}

- Heating and cooling equipment will still be somewhat bulky. 
- Large diameter nitrogen lines will be required due to the use of low pressure nitrogen.

\section{DETALS OF ITCAP}

\section{Column fabrication}

The column design can be similar to present stainless steel coil TCAP units. The basic components include a stainless steel coiled column filled with $\mathrm{Pd} / \mathrm{k}$, a shell (jacket), and a cylindrical baffle. The coil occupies the annular space which is formed between the shell and the baffle. Hot or cold nitrogen is circulated through the annular space to heat and cool the column alternately to effect the separation of hydrogen isotopes. A schematic of the column design and the associated equipment are shown in Figure 3.

There are three major improvements in the column design: (1) increased heat transfer in the $\mathrm{Pd} / \mathrm{k}$ column, (2) decreased tritium permeation, and (3) reduced heat load of the heating and cooling system.

Heat transfer in the $\mathrm{Pd} / \mathrm{k}$ column has been a limiting step for the $\mathrm{H \& C}$ process which negatively impacts cycle time. Heat transfer in the $\mathrm{Pd} / \mathrm{k}$ column can be improved by adding aluminum foam. It has been shown in the on-board hydrogen storage system of the H2Fuel bus that the use of aluminum foam in a hydride bed improved the hydride heat transfer coefficient by a factor of 5 . Using the aluminum foam in the $\mathrm{Pd} / \mathrm{k}$ column is expected to have a similar effect and should be evaluated. Aluminum foam densities range from $3 \%$ to $12 \%$ depending on type and a range of $5 \%$ to $7 \%$ is recommended. If desired, the space occupied by the aluminum foam can be compensated for by increasing the diameter of the column.

Tritium permeation calculations indicated that TCAP columns can permeate several hundred curies per year to the H\&C nitrogen system. To limit the build-up of tritium in the H\&C nitrogen, a means to remove the tritium will be required. The same calculation showed that if a continuous nitrogen purge is used to control the tritium concentration, a purge rate of several SCFM will be required to keep the tritium concentration below $0.01 \mathrm{Ci}$ /liter. This purge of nitrogen can be directed to the glovebox nitrogen system and should not adversely affect the glovebox nitrogen purge system. The tritium concentration can be further reduced if a layer of aluminum, or some other type of permeation barrier, is coated on the surface of the column. Ways to make the aluminum coating and its effectiveness should be investigated.

The thermal swing of the column's jacket (and ends) is a significant H\&C load to the TCAP. With the addition of thermal insulation to the inside of the jacket and the inlet piping, the heat load of the jacket and the inlet piping, which can account for more than $50 \%$ of the total heat load, can be significantly reduced. The reduction of column's heat load will permit the use of smaller H\&C equipment.

\section{Heating and cooling rate}

For a fixed ITCAP heat load, the mass flow of H\&C nitrogen should be constant and is proportional to the product of pressure times volumetric flow rate (linear velocity for pipe of constant crosssections). For a high pressure nitrogen system, lower linear velocities are needed than for atmospheric pressure nitrogen. For atmospheric nitrogen, higher linear velocities are needed, but these velocities can be made to be less than $10 \%$ of the sonic velocity. Higher velocity, lower pressure nitrogen will permit the use of blowers instead of compressors, and will greatly reduce the potential of contaminated nitrogen leaking from the piping to the ambient.

The lower pressure nitrogen should not affect the external coil heat transfer rate provided the mass flow rate is the same. This is based on the heat transfer equation for a gas flowing through a band of 


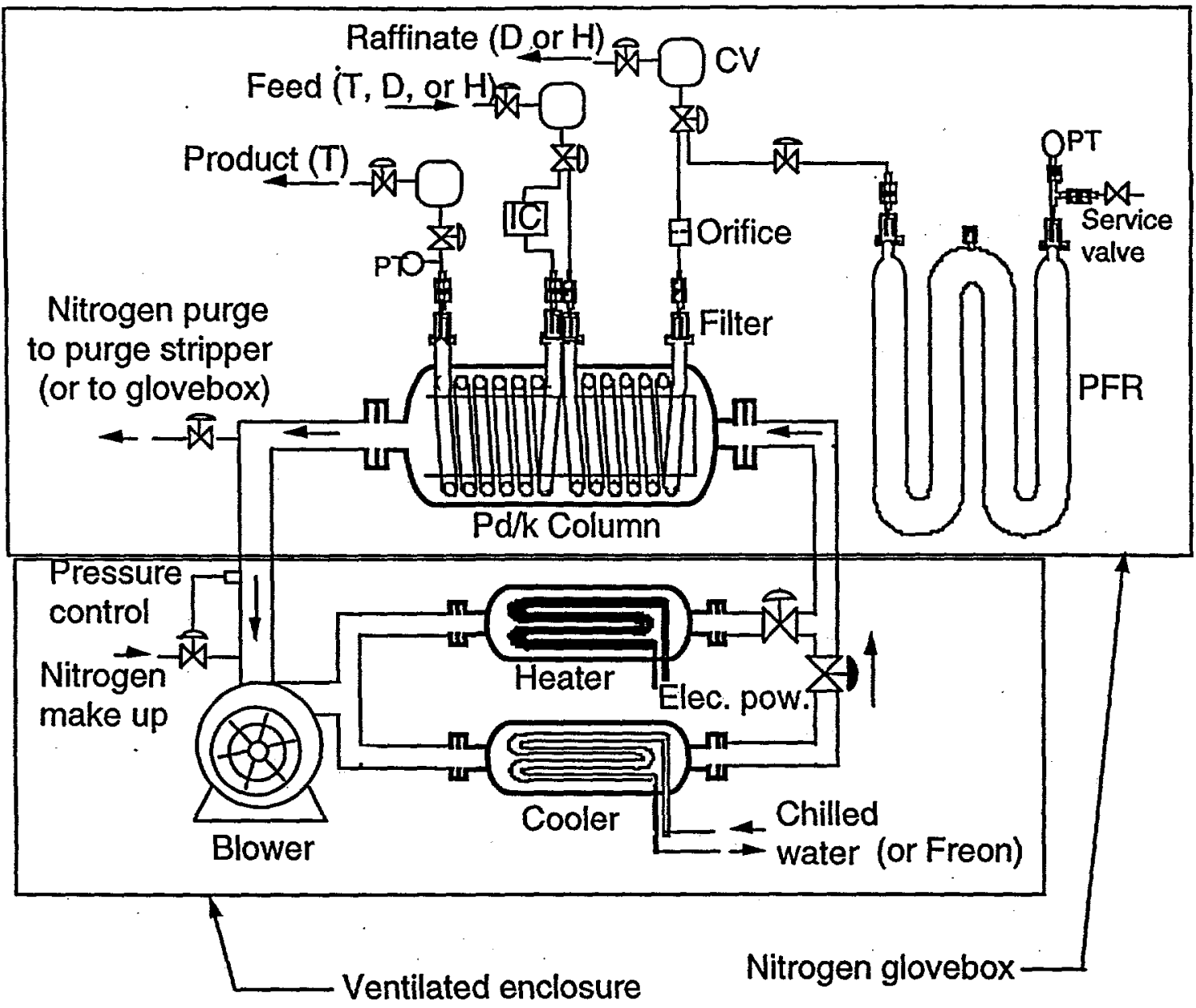

Figure 3. Improved TCAP Hydrogen Isotope Separation System

in-line tubes (present column design). The heat transfer coefficient between the $\mathrm{H} \& \mathrm{C}$ nitrogen and the column wall is dependent upon the mass rate and not dependent upon pressure (Ref. 2):

$$
\begin{aligned}
& \frac{h_{m} D_{0}}{k_{1}}=0.26 P^{03}\left(\frac{G_{\max } D_{0}}{\mu_{i}}\right)^{0.6} \\
& \text { where } \quad h_{m}=\text { heat transfer coefficient, Btu/hr } / \mathrm{ft}^{2} /{ }^{\circ} \mathrm{F} \\
& \mathrm{D}_{\mathrm{O}}=\text { outside diameter of the tube, } \mathrm{ft} \\
& \mathrm{kf}=\text { thermal conductivity of } \mathrm{gas}, \mathrm{Btu} / \mathrm{hr} / \mathrm{ft} /{ }^{\circ} \mathrm{F} \\
& \mathrm{Pr}=\mathrm{Prandtl} \text { number of gas, dimensionless } \\
& \mathrm{G}_{\text {max. }}=\text { maximum mass flux, Lb. } / \mathrm{sec} / \mathrm{ft}^{2} \\
& \mu_{\mathrm{f}}=\text { viscosity of gas, } \mathrm{Lb} . / \mathrm{ft} / \mathrm{sec}
\end{aligned}
$$

For a constant mass flow rate of gas reduced to a pressure of 1 atmosphere, the value for $\mathrm{h} \mathrm{m}$ is approximately the same as for higher pressure gas since the values for $\operatorname{Pr}$ and $\mu_{\mathrm{f}}$ do not change significantly with pressure. For a new column design using aluminum foam, as discussed above, it is estimated that the internal heat transfer coefficient will be increased by a factor of 5 , and the overall heat transfer coefficient will be increased by at least a factor of 3 . 
With the heat transfer correlation, a blower for 800 SCFM can be made to yield the same heat transfer coefficient as a 10-atm compressor. Using equations for pressure drop calculations (Ref. 3), the column pressure drop was estimated to be less than 1 PSI.

\section{Heat load Calculations and Equipment Sizes}

For a 5 minute heat-up (or cool-down) time, the heat load is $100,000 \mathrm{Btu} / \mathrm{hr}$ (nominally $30 \mathrm{~kW}$, or 8 tons, or $40 \mathrm{hp}$ ), which allows for excess capacity. The use of heat ballast can reduce the size of the refrigeration unit and the size of the heat exchangers, but will probably increase the number of the blowers. Their use in the H\&C systems should be evaluated.

The heat loads for one TCAP unit are estimated and listed below:

\section{fraction of total}

$\begin{array}{lll} & \text { Coiled Tube } & 0.20 \\ \text { Pd/k } & 0.09 & \\ \text { Abs./des. heat } & 0.06 & \text { (averaged on } 150^{\circ} \mathrm{C} \text { swing) } \\ \text { Jacket \& ends } & 0.46 \\ \text { Total } & & 0.20 \\ \text { H\&C Piping } & 1.00\end{array}$

\section{ALTERNATIVE TCAP DESCRIPTION: RABIT TCAP}

The alternate TCAP design developed by Scogin, Howard, and Lee has been referred to by several names based on the general characteristics of the columns design described below. The name "Rectangular Array of Bored in Tubes" (RABIT) TCAP is desired since the name in itself is very descriptive of the column features, does not include the type of material in its name, and does not contain the word "block". Some names used include "Swiss Cheese" TCAP which describe the appearance of the column when viewing the top or bottom projection drawing of the column, "Copper Block" TCAP for when machining a block of copper for fabrication was proposed. The name "Rectangular Array of Bored in Tubes" (RABIT) TCAP was chosen because "Swiss Cheese" was not very descriptive of the column's other (non-top/bottom view) features and references to name including "block" were dropped to reduce (and hopefully eliminate) confusion and reference to the original RTF TCAP column design which was the "Aluminum Block" TCAP.

\section{RABIT TCAP SUMMARY}

- Holes drilled into a copper block form a serpentine column.

- Eliminates all nitrogen based H\&C.

- Electrically heated.

- Liquid (water/glycol) cooled.

\section{RABIT TCAP ADVANTAGES}

- Has small glovebox volume.

- Has no H\&C external to the glovebox.

- Fast heat transfer with Aluminum or copper foam

- Greatly reduces permeation by using heavy walled copper.

- Has much simpler support system.

- Conservative, robust technology used for heating and cooling. 


\section{RABIT TCAP DISADVANTAGES}

- Unproven design.

- Potential for boiling coolant.

- Method of attaching plugs has not yet been selected.

\section{RABIT TCAP CONFIGURATION}

\section{Motivation for the Configuration}

In the conventional TCAP design, most of the heat that must be cycled into and out of the TCAP apparatus goes not to changing the temperature of the sorbent, or to the heat of sorption of the hydrogen, but, instead, to changing the temperature of the 'structure', i.e., the tubing containing the sorbent, the jacket surrounding the coolant, the internal baffle, etc. Therefore a reduction in the heating/cooling duty could be achieved by reducing the thermal inertia of these 'structural' elements, either by reducing their mass, or by reducing their specific heat, i.e., their heat content per unit mass. This goal of reducing the thermal inertia of the structure motivated the design of the Rectangular Array of Bored in Tubes (RABIT) TCAP configuration.

Simplicity of heating and cooling was the second goal of the RABIT TCAP. Direct contact electrical resistance heating was chosen for the heating method, and circulating chilled water was chosen for the cooling method. Glycol as a cooling media is also a possibility, due to its greater operating temperature range than water, if concerns over potential contamination and waste issues can be resolved.

\section{Basic Design Parameters}

Because metal foam (either aluminum or copper), which is estimated to occupy $5 \%$ to $7 \%$ of the column volume, is planned to be inserted into the sorbent column to increase heat transfer, the diameter of the column can be increased by $2.5 \%$ to retain the same cross sectional area available for flow with-respect-to a column without foam. With the assumption of feeding the RABIT TCAP at the center of the column, the column volume can be divided into two modules (rectangular arrays), one for stripping and one for rectification.

Computer simulation of TCAP performance indicates that operating the column at a coolant temperature compatible with chilled water coolant may require as much as $40 \%$ additional column length compared to a Freon based cooling system, but this requires experimental verification. In the event additional column length is needed, the RABIT TCAP can either add more modules or use larger modules with more holes to obtain the required length.

\section{Column Fabrication}

Each module consists of an array of 12 to 18 parallel bored in tubes or channels. The channels will be connected alternately at their ends to achieve a serpentine, or zigzag, flow path for the hydrogen gas flow.

Using electrical heating and chilled water/glycol cooling requires that the material of construction have a high thermal conductivity to transfer heat along a somewhat lengthy and tortuous path without having an unacceptable temperature gradients. Copper was chosen as the basic material of construction. For compatibility with hydrogen exposure, the highest purity oxygen-free copper is required: Copper-101 (preferred) or Copper-102. Aluminum was considered but eliminated for several reasons: primarily due to concerns with corrosion in the cooling channels and with overaging the aluminum alloy.

The wall thickness of the sorbent channels are governed by the requirement of withstanding the anticipated pressure at the high temperature operating condition. The specified design conditions 


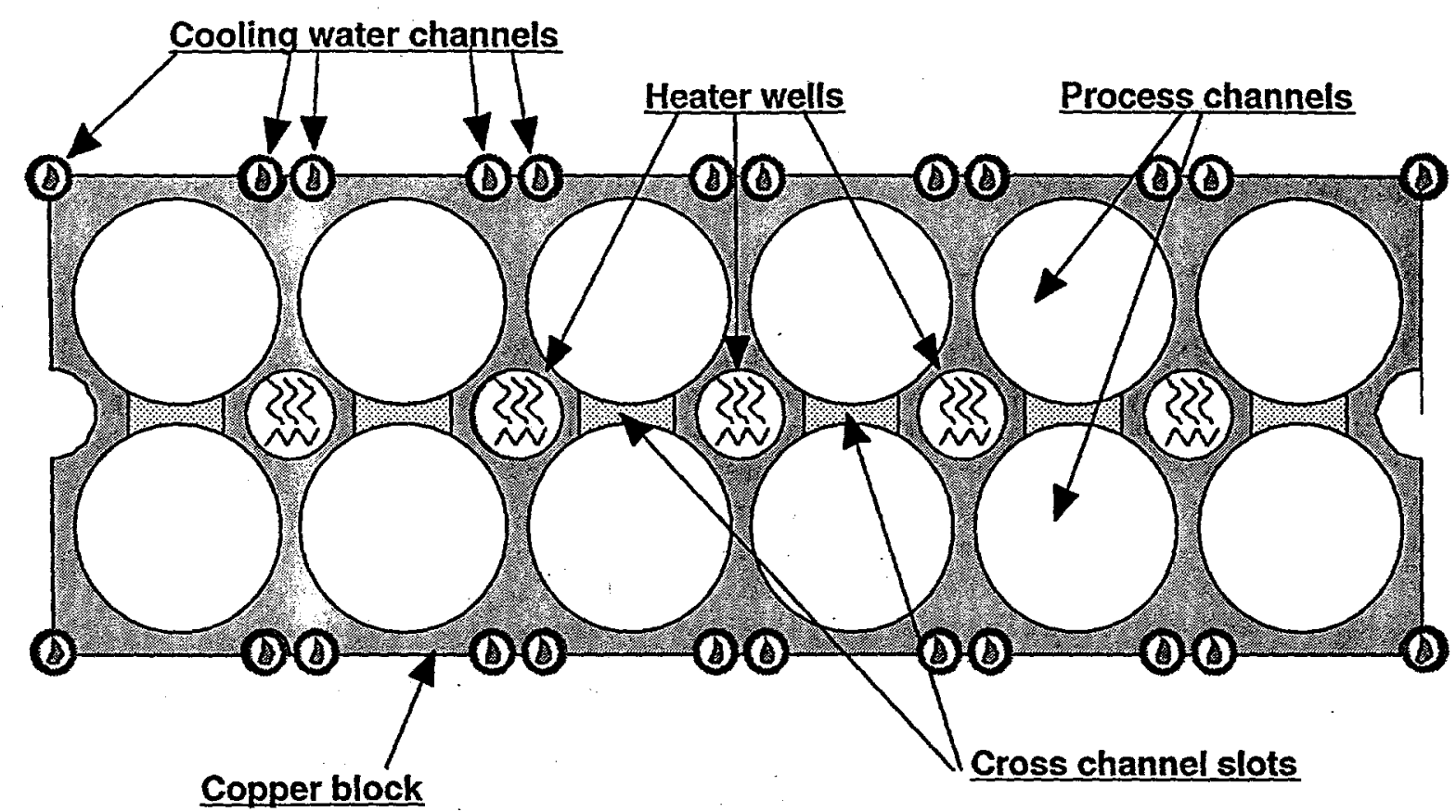

Bottom view

Figure 4. RABIT TCAP Column Cross-Section at Bottom

were for 300 PSIG at $200^{\circ} \mathrm{C}$. The RABIT TCAP was designed using ASME codes to withstand 500 PSIG at $400^{\circ} \mathrm{F}$, which requires a wall thickness of 0.096 inches for Copper-101. Thermal inertia is reduced by having adjacent parallel tubes share a common wall, effectively reducing the required wall thickness in half. The exterior walls of tubes, which separate sorbent columns from coolant channels, from heater wells, or from the environment, are required to be full thickness, so the sorbent column wall thickness cannot be completely reduced in half. Additionally, manufacturing tolerances require a membrane somewhat thicker than this minimum.

The sorbent column channels are manufactured by boring or drilling an array of 12 to 18 holes (tubes) into a copper block. The hole centers are located on a 2 wide rectangular array. Connections at the ends between channels are achieved by milling a slot between alternate adjacent channels at each end of the block. The ends of channels, and the slots, are closed by a 'dumbbell'-shaped plug, which fits into the two adjacent channels and their connecting slot, and is either welded or brazed in place.

\section{Heat Transfer}

Electrical heating will be supplied by 5 cartridge heaters in each module, based on a twelve tube design. The heat load was determined by requiring the modules to heat their minimum temperature to their maximum temperature in 5 minutes. Each cartridge heater will supply $1.5 \mathrm{~kW}$, for a total of $15 \mathrm{~kW}$, allowing for an excess heating capacity. The heating cartridges are a nominal $3 / 8$ inch diameter, slide into wells of 0.406 inch diameter which are located at the mid-point of the sorbent channel lattice diagonals. This gives a thickness for the membrane between sorbent channel and heater well of 0.120 inches, equal to the membrane thickness between sorbent channels.

Cooling is accomplished by circulating chilled water through $1 / 4$ inch diameter copper tubes which are brazed to the exterior surfaces of the copper block. The cooling is divided among 4 parallel 


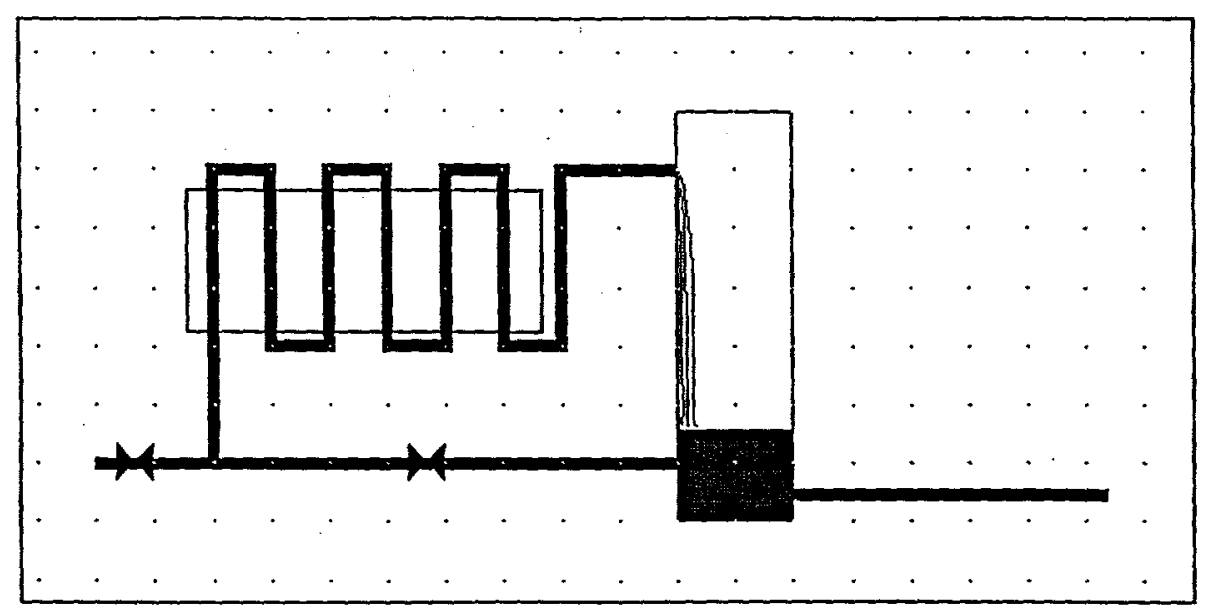

Figure 5. RABIT TCAP Cooling Flow

circuits, each circuit making 6 passes in series in contact with the block. The water flow in each circuit starts from a common header at the bottom of the block, flows through the 6 passes on the block, completes a 7th pass to join a header at the top of the block and then exits to a collection reservoir. To ensure proper drainage of the tubes by natural siphon, 1/4" cooling tubes are specified.

The total cooling duty required to cool both modules from their maximum temperature to their minimum temperature in 5 minutes is about 4.5 tons of refrigeration. Assuming a chilled water temperature of $10^{\circ} \mathrm{C}$, the total chilled water flow to both modules is $6 \mathrm{gal} / \mathrm{min}$. to provide excess cooling capacity.

The heat transfer coefficient in the sorbent channel is assumed to be increased by a factor of 5 with the use of metal foam over values without the foam. Copper foam, which is expected by the manufacturer to be on the market within a few months, should be even more advantageous; however; the RABIT TCAP design assumes that only aluminum foam is available.

Figure 4 shows a ("Swiss Cheese") cross-section of the block at the bottom to illustrate the location of the sorbent channels, the connecting slots, the heater wells, and the channels into which the coolant tubes are brazed. A cross-section at the top of the block would be similar except that the connecting slots would be cut in the longitudinal direction instead of the transverse direction, and would join alternate pairs of process channels to those connected at the bottom.

The cooling circuit supply is also connected by a drain valve to the bottom of the same collection reservoir into which the cooling circuits discharge. The forward flow through the cooling circuits discharges into the upper head space of the collection reservoir, while the drain valve discharges into the bottom of the collection reservoir. When the cooling cycle ceases, the supply of cooling water to the circuits is shut off, and the drain valve is opened allowing the cooling circuits to drain back into the collection reservoir by siphon action. Draining the cooling tubes at the end of the cooling cycle, prevents the consumption of heat by evaporating or heating the water during the subsequent heating cycle. Figure 5 illustrates the arrangement of the cooling circuit, valves, and collection reservoir. The cooling circuit tubing is sized such that the multiple loops of the cooling circuit can empty by siphon action, yet still achieve adequate flow. If the coolant tubes are too large, they will not siphon properly, but leave liquid behind in the bottom of each loop; if they are too small, they will not pass adequate coolant flow without an excessive pressure drop.

The modules will be housed within a vacuum jacket to minimize heat exchange with the environment during the temperature cycles. Only a rough vacuum, approximately a few torr pressure, will be necessary to achieve adequate thermal insulation. 
Protection against overheating will involve multiple cascaded interlocks. The heaters will be controlled through a controller which will have a High-Alarm interlock set $10^{\circ} \mathrm{C}$ above the maximum operating point, which will automatically kill all power to the heaters. A High-HighAlarm interlock will automatically restart the cooling water flow at $15^{\circ} \mathrm{C}$ excess temperature. A High-High-High-Alarm interlock at $20^{\circ} \mathrm{C}$ excess temperature will stop vacuum exhaust of the jacket, flood the jacket with nitrogen, and start cooling water flow attached to the jacket. Finally, at $25^{\circ} \mathrm{C}$ excess temperature, a fusible link in the heater electrical supply, mounted physically on the copper block, would melt and interrupt power. Even if all of these protective measures were to fail, the maximum temperature of the copper block is estimated to still be below the melting point of the aluminum foam, and well below the melting point of the copper, even assuming heat transfer from the block to the jacket only by radiation, and heat loss from the jacket to the surroundings only by radiation and natural convection. If copper foam is used instead, an even greater safety margin exists.

\section{TCAP SYSTEM DESCRIPTION}

The tritium enrichment and hydrogen enrichment functions of the Consolidation Project will most 'easily be performed by the same system. The system will consist of four TCAP units of equal size and four separate sets of storage beds. Unless otherwise specified, all piping in the TCAP system will be rated for 500 PSIG.

\section{TCAP UNIT}

A single TCAP unit consists of a column in two sections, a PFR, three calibrated volumes, instrumentation, and the associated piping and valving (Figure 6).

All columns will have instrumentation as shown in Figure 6 . The four columns will all be $2 x$ size. This size is driven by the following assumptions: 1) two columns dedicated to protium enrichment, 2) an attainment of $70 \%, 3$ ) cycle times $\leq 40$ minutes, and 4) a temperature swing of $25^{\circ} \mathrm{C}$ to $150^{\circ} \mathrm{C}$. A temperature swing of $-20^{\circ} \mathrm{C}$ to $150^{\circ} \mathrm{C}$ is recommended to provide some contingency. The team felt this contingency was necessary so the columns would meet the project's separation needs in the event additional gas volumes were to be processed or the column's operation was inadequate to meet the protium purity levels for release from the facility.

\section{Column}

The column is divided into two sections, with the feed point located between them. (The sections need not be of the same size.) Flow in the column and PFR must not undergo unnecessary mixing; 3port, 2-way valves are used to minimize dead volume and eliminate tees.

The $\mathrm{Pd} / \mathrm{k}$ in the column sections is contained by screens; sintered metal filter fingers are used to keep fine metal hydride particles out of the system piping.

In order to maintain and calibrate the pressure transducers on the column, a special valving arrangement has been designed. The through leg of a 3-port 2-way valve is placed in-line with column, and the bellows section connects through a second 3-port 2-way valve to the pressure transducer. A service header is connected to the bellows port of the second 3-port 2-way valve. During normal operation, the only additional volume added to the system is the one bellows of the in-line 3 port 2-way valve.

\section{PFB}

The plug flow reverser (PFR) is an essential part of a TCAP unit. The flow into and out of the PFR must be laminar. The PFR is usually implemented as a packed column with a large void space and a 


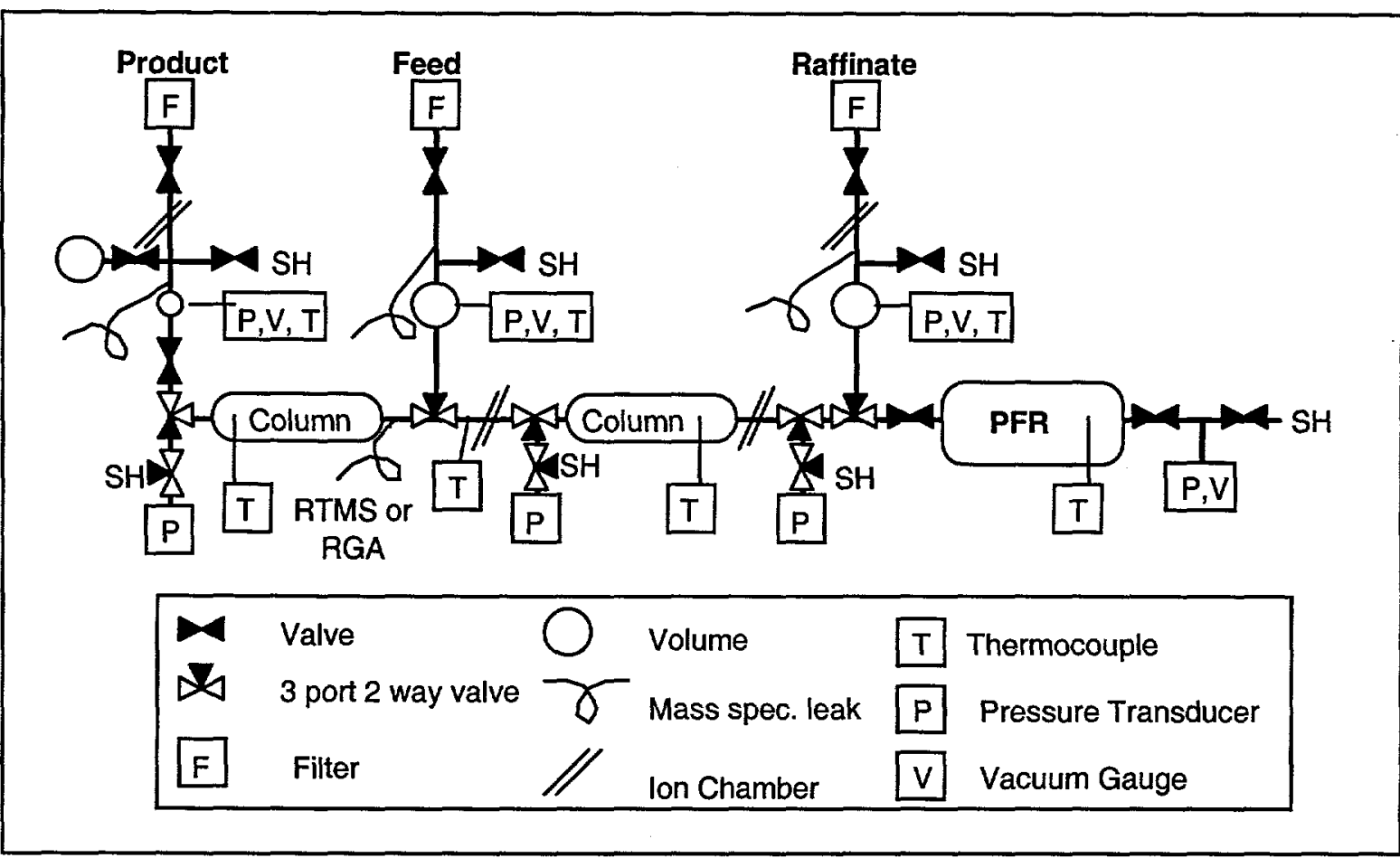

Figure 6. Generic TCAP Unit Drawing

low pressure drop. Currently, the PFR is implemented as a 4 " pipe filled with $\mathrm{Pd} / \mathrm{k}$. The nominal PFR volume should $1 / 2$ the nominal column hydrogen capacity.

$\underline{\text { CVs }}$

Calibrated volumes (CVs) are used to meter the feed into the column and to meter the product and raffinate streams out of the column. The feed and raffinate CVs are connected to the column by 3port 2-way valves to reduce mixing in the column. All CV sections generally have the same instrumentation and connections: a pressure transducer, a thermocouple, a TCVG, a mass spectrometer leak, and a service header connection. The $C V$ sections also have an exit valve which is used to define the volume of the section. There is a filter at this valve, which slows the flow out of or into the $\mathrm{CV}$ so that the pressure can be accurately controlled. The product and raffinate CVs also have in-line ion chambers which will be used to determine to which bed the outputs should be directed.

\section{BED UNITS}

The TCAP system will require four (five if deprotiating deuterium) sets of hydride storage beds: 1 ) pure tritium $(\mathrm{T}) ; 2)$ medium concentration tritium, $>10 \%(\mathrm{M}) ; 3)$ low concentration tritium, $<10 \%(\mathrm{~L})$; 4) stackable gas (S), and optionally, 5) deprotiated gas(D). The medium and low concentration beds will normally provide feed to the columns, but will also take out-of-spec gas intended for the tritium and stackable beds.

The tritium and stackable beds will have at least two independent units, one for absorbing gas from the TCAPs using the low pressure header, and one in transition or in service elsewhere. The medium and low concentration beds must have at least three independent units, one for absorbing from the low pressure header, one for feeding to the high pressure header and one in transition or in 


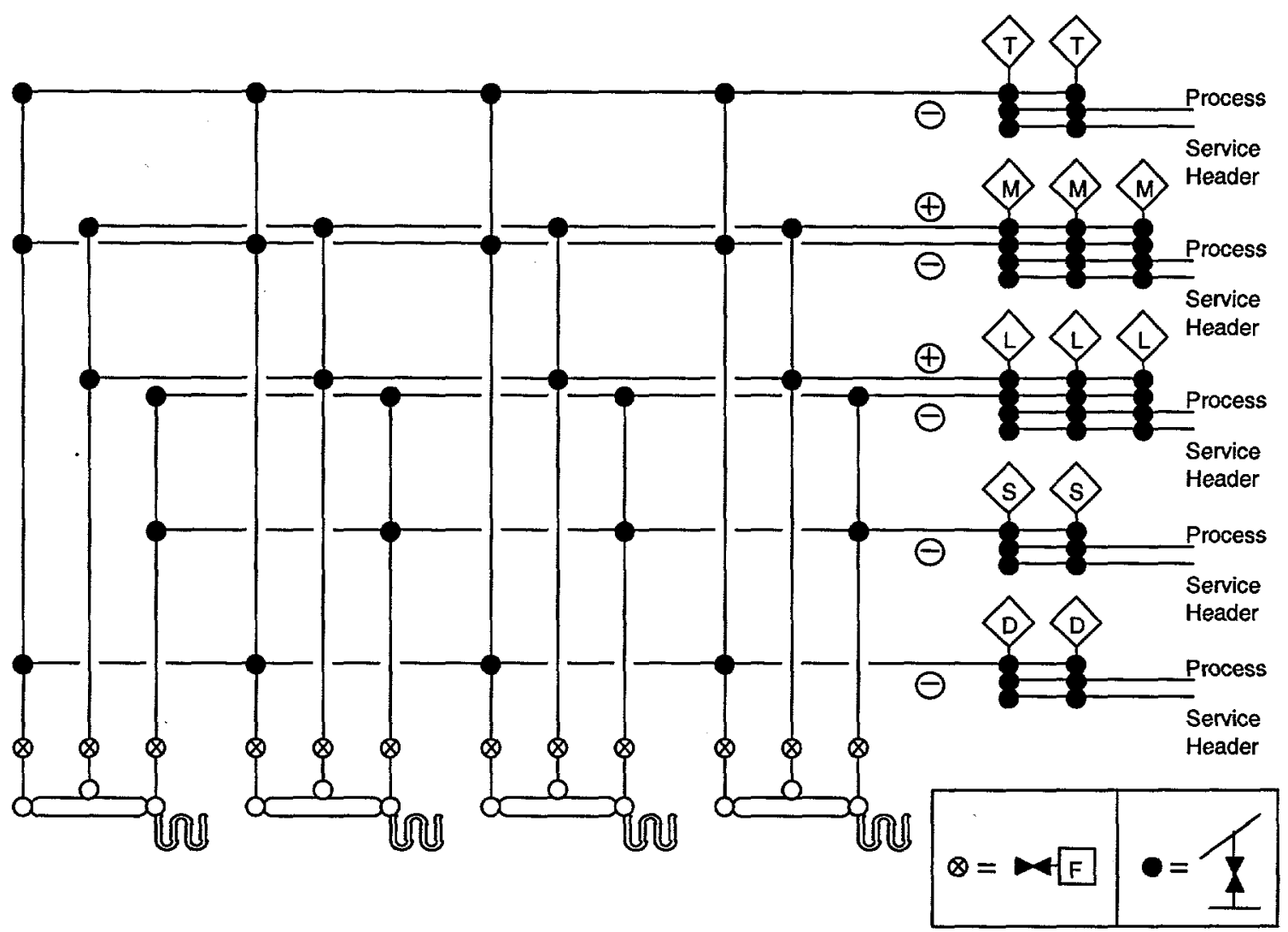

Figure 7. TCAP/Hydride Bed/Header Process Piping

service elsewhere. If deprotiated deuterium is produced, at least one bed must be installed for this operation.

\section{HeAders}

Each bed within a set will be connected to three or four common headers. All sets will have a process header for connections with other systems, a service header for gas transfer within the isotope separation system and for maintenance, and a low pressure $(-)$ header for receiving gas from the TCAP units. The medium and low concentration beds will also have a high pressure $(+)$ header for feeding the TCAP units.

\section{TCAP to Bed connections}

Each TCAP has identical connections to the beds (Figure 7). In practice, one TCAP unit may be designated to process medium concentration feed to produce high purity tritium, two TCAP units may process low concentration feed to produce stackable gas, and one TCAP will be available for backup.

Each CV will have a header which will connect to one or more feed bed header. Each product CV will be connected to the low pressure header of the tritium and medium concentration beds (and optionally to the deprotiated bed). The feed CVs will be connected to the high pressure headers of the medium and low concentration beds. The raffinate CVs will be connected to the low pressure headers of the low concentration and stackable beds. 


\section{$\underline{\text { Service Headers }}$}

The service header is provided for maintenance, calibration, evacuation, gas transfers, and inerts removal within the isotope separation system. The service header should be connected to both the flush gas and production evacuation systems. Inert gas removal is an essential function, the TCAP units must have $\leq 0.1 \%$ inerts (non-hydrogen gas).

\section{Other Services}

- A dedicated vacuum system will be required to provide vacuum for the insulating vacuum jackets.

- Mass spec leaks must be provided for each TCAP(4) and each bed unit (1).

- Stripper for glovebox

- Cooling water and electric heating to heat and cool the TCAP units, and H\&C for the hydride beds

- Control and data acquisition capabilities.

\section{ReCOMmendations/Path Forward}

Based on comments from the Pre-Conceptual Design Review Team, a dual track TCAP development program should be initiated.

The first and highest priority is to demonstrate the Improved (SS Coil) TCAP design as the reference design for the Tritium Consolidation Project. Since this unit may be similar in design to the current RTF TCAP, most of the development will be aimed at demonstrating heating and cooling methods and hardware as well as possible tritium permeation barriers.

The second priority will be to continue to develop the RABIT TCAP with Process Development funding. Due to its size and cycle time advantage, this unit will be selected as the candidate unit for testing at LANL (if funding is made available). The "hot" RABIT TCAP tests at LANL will provide confirmatory TCAP purity and capacity information which can be directly related to the performance of the Improved (SS coil) TCAP.

The RABIT TCAP unit will be the backup unit for the Consolidation Project. Should there be any project delays and sufficient time and advantages exist to change TCAP designs the RABIT TCAP unit can be used to replace the Improved (SS Coil) design. In addition, the RABIT unit will become the eventual replacement for the RTF units since RTF space limitations will preclude the use of the Improved TCAP design.

A detailed development plan that reflects the dual path approach is being developed and is expected to be completed early in FY98. Some activities to be included in this plan are 1) initial heat transfer tests using "short" $\mathrm{Pd} / \mathrm{k}$ filled tubes to measure heat transfer coefficients for a variety of tubing types/parameters, 2) a fabricability demonstration of the column/unit, 3) assembly of a representative heating and cooling system (chilled water temperature to $150^{\circ} \mathrm{C}$ ) with blowers, valves, piping, and hot and cold sources/heat exchangers, 4) heat transfer cycling tests of the demonstration unit, 5) and tritium permeation tests.

\section{REFERENCES}

1. TCAP Technical Requirements for the Tritium Facility Modernization and Consolidation Project, S-7726, WSRC-RP-97-00939, 1997.

2. William H. Adams, "Heat Transmission", 3rd edition, p272.

3. Frank Kreith, "Principles of Heat Transfer", 2nd edition, p380-424. 